\title{
Self-removal of long-acting reversible contraception: A content analysis of YouTube videos
}

Kathleen Broussard ${ }^{\mathrm{a}}$ and Andréa Becker ${ }^{\mathrm{b}}$

apopulation Research Center, University of Texas at Austin

305 East 23rd St

Austin, TX 78712

${ }^{\mathrm{b}}$ The Graduate Center, CUNY

3655 th St

New York, NY

Corresponding author:

Kathleen Broussard

$\underline{\text { Rkbroussard@prc.utexas.edu }}$

Andréa Becker

abecker@gradcenter.cuny.edu

\section{Declarations of interest: none}

Funding: This research was supported by grant P2CHD042849, Population Research Center, awarded to the Population Research Center at The University of Texas at Austin by the Eunice Kennedy Shriver National Institute of Child Health and Human Development. The content is solely the responsibility of the authors and does not necessarily represent the official views of the National Institutes of Health. This research is also supported by the National Science Foundation Graduate Research Fellowship Program under Grant No. 2018259906. Any opinions, findings, and conclusions or recommendations expressed in this material are those of the author(s) and do not necessarily reflect the views of the National Science Foundation. 


\begin{abstract}
Objective: To explore publicly available information about the self-removal of long-acting reversible contraception (LARC) on a popular video-sharing website.
\end{abstract}

Study Design: We conducted a comprehensive keyword search of YouTube videos related to selfremoval of long-acting reversible contraception (LARC) - namely intrauterine devices (IUDs) and implants. We analyzed video content to explore demographic characteristics, method and duration of LARC use, and motivations and experiences of self-removal.

Results: Our keyword search identified 58 videos that met the criteria for inclusion, including 48 videos that featured individuals who removed an IUD and 10 who removed an implant. Collectively, videos had over 4 million views. We identified most video creators as white (53.4\%), 31\% as Black, and $14.3 \%$ as Latinx. Reasons for LARC discontinuation included negative side effects, fear of potential side effects, and desire for pregnancy. Users were motivated to remove their own device by both preferences and barriers to formal care. Fifty-six individuals (96.6\%) successfully removed their device, and most described their experience in positive terms related to the ease of removal.

Conclusion: This study builds upon prior research by describing publicly available information about LARC self-removal. The over-representation of Black women in our sample may reflect a higher prevalence of LARC self-removal among this population. Positive experiences of selfremoval and high levels of viewer engagement with online videos suggest a greater need for provider counseling on LARC removal at the time of insertion.

Implications: Prior to LARC insertion, patients should be made aware of any financial requirements for discontinuation. Provider counseling for self-removal at the time of insertion will likely minimize health risks and affirm patient reproductive autonomy.

Keywords: Self-removal; IUD; implant; online; contraceptive attitudes; discontinuation 


\section{Introduction}

Over the last 20 years, the proportion of contraceptive users turning to long-acting reversible contraception (LARC) in the United States is steadily rising; 14\% were using an intrauterine device (IUD) or contraceptive implant in 2018 compared to just $2.4 \%$ in 2002 [1,2]. This trend has occurred in tandem with dramatic declines in reproductive healthcare access in the United States, including a change in Title X regulations that reduced the number of family planning providers [3], restrictive anti-abortion policies that have led to the closure of clinics that provide contraception and abortion services [4,5], and the new challenges of sexual and reproductive healthcare access during the COVID-19 pandemic [6,7]. Notably, since the beginning of the pandemic, the American College of Obstetrics and Gynecology has advised their members to continue offering insertion of LARC "where possible" but to "postpone routine LARC removals, if possible, and counsel LARC users on the effectiveness of extended use beyond the labeled duration" $[8,9]$.

Given the presence of barriers to clinic-based LARC removal, we build on two recent studies that explore public interest and experience with the self-removal of intrauterine devices $[10,11]$. Extant knowledge on this topic is limited. One study found that people discussing selfremoval on websites and online forums experienced barriers to clinic-based removal [10]. Another study invited women seeking provider-removal to attempt self-removal in the clinic setting; roughly half of patients were unwilling to attempt self-removal and only $20 \%$ of those who attempted were successful [11]. In contrast, we are not aware of any existing research on the self-removal of contraceptive implants. Given the parallel increase in use of implants and even greater barriers to a desired discontinuation, further inquiry of people's experiences seeking removal is needed.

Due in part to these racialized perceptions and experiences with birth control, researchers have also examined racial patterns regarding the self-removals of LARC. Historian Dorothy Roberts (1999) has written about women of color, particularly Black women, feeling misled or misinformed 
by doctors who did not explain the cost of discontinuing LARC, leading to self-removal at home [12]. In other cases, doctors refused to remove LARC before its expiration date, likewise leading to self-removals (ibid). Contemporarily, Foster et al. (2014) found that Black women were particularly interested in self-removing their IUD when invited to do so in a clinic setting, though most participants, regardless of race, were unsuccessful in removing their device [11].

In this study, we contribute to this nascent body of research by analyzing publicly available narratives of IUD and implant self-removal on YouTube. People increasingly rely on the internet as a primary source of health information $[13,14]$. As part of this trend, researchers have begun to investigate people's health-seeking strategies and the information available online for a wide range of reproductive health needs $[10,15-20]$. An analysis of videos on YouTube allows us to examine the demographic characteristics of people who attempt self-removal of LARC outside of the clinic setting — data that was not available in previous studies [10,11,21]. While extant work is limited to studies of IUDs, our data is novel in that we include experiences of implant self-removal. Although the self-removal of implants is likely less prevalent than the self-removal of IUDs, the large number of video contributors on YouTube allows us to explore determinants and experiences of both phenomena. Importantly, our analysis grants insight into the information and narratives people may encounter about self-removal while searching online, including who self-removes, why they wish to discontinue LARC and remove their device at home, and the quality and outcome of their experiences. These portrayals likely shape would-be-patient decisions and are therefore critical for informing best practices of clinic-based counseling at the time of insertion.

\section{Material and Methods}

\subsection{Sample and search strategy}

We produced a comprehensive list of YouTube videos using two separate keyword searches related to the self-removal of contraceptive implants and intrauterine devices (see Appendix A for 
keyword search terms). All videos were compiled from January-March 2021. To prevent the influence of algorithm-based results dependent on the authors' personal search histories, we used an incognito window to conduct all searches. Videos were excluded from our analysis if they (1) only portrayed clinic-based LARC removal, (2) did not include a person on-screen who considered or attempted self-removal, (3) were recorded in languages other than English, or (4) were otherwise unrelated to our inquiry. The final sample size was determined with a discontinuation rule of 50 videos; when 50 consecutive videos of each keyword search did not meet our eligibility criteria, no further videos were included.

\subsection{Analysis}

A coding guide was designed to capture both quantitative and qualitative video content. We divided the dataset in half and separately recorded the characteristics, motivations, and experiences of people who removed their own IUDs and implants. For each video, we recorded the number of views, number of "likes" and "dislikes", and number of viewer comments. Additionally, we documented the top two viewer comments for each video. Top comments are those which are visible directly below the video; they rise to the top of the comments section through an algorithm that considers factors such as the date it was posted, the number of likes or dislikes it has received, and the number of user responses to each comment.

To assess the demographic characteristics of video subjects, each author separately recorded their perceptions of the subject's race and ethnicity, age group, and gender. Although this method of attribution is imperfect and may fail to reflect how an individual self-identifies in terms of race or gender, the aim of this approach is to describe the characteristics of video subjects as they are likely perceived by viewers on YouTube. We had high intercoder reliability, recording similar demographic characteristics in $94.8 \%$ of cases. In the few instances when our perceptions differed, we conducted a second review of videos together to reach agreement. We also wrote detailed memos while 
watching each video. These memos included information about subjects' perceptions and attitudes towards their LARC device, reasons they wished to discontinue LARC, why they attempted removal at home, and their experiences of self-removal. To analyze this qualitative content, we developed a coding scheme based on themes and patterns that emerged during our first round of observations. We then assigned codes to our recorded memos for each video.

This study did not involve human subjects and was deemed exempt from requiring approval by the Institutional Review Board at The University of Texas at Austin.

\section{Results}

Our final sample includes 58 videos that portrayed people's experiences with self-removal of an IUD or implant. Table 1 presents the characteristics of video subjects for the total sample, and separately according to LARC method type. Approximately $83 \%(n=48)$ of videos depicted the selfremoval of an IUD, while the remainder $(n=10,17.2 \%)$ showed the self-removal of a contraceptive implant. Videos featuring IUD self-removals differed from those featuring implants in important ways. All IUD users had already attempted self-removal prior to recording their videos, except one woman whose partner removed her IUD in real-time off-screen. In contrast, 9 out of the 10 video subjects with implants performed a live, on-screen removal of their device. We identified 57 individuals as women and one as non-binary. Over $90 \%(n=53)$ of video creators were between the ages of 20-39, with most $(n=39)$ in their twenties. This age distribution was similar across IUD and implant users. Most video creators were white $(n=31)$, however, almost a third of our sample was Black (33.3\% of IUD users and 20\% of implant users). We identified 8 women as Latinx (13.8\%), and one woman as Native American (3.4\%). One woman who removed an implant never showed her face on-screen, so her race/ethnicity and age in Table 1 are coded as 'Unknown'.

Table 1 also describes the subject's previous childbearing, method of LARC, and duration of method use before attempting self-removal. Among the full sample, 22 (37.9\%) explicitly mentioned 
a previous pregnancy or existing children. Among those who attempted IUD self-removal, 70.8\% had a hormonal method and 29.2\% had a non-hormonal, copper IUD. Although 6 subjects (12.5\%) did not report how long they had been using an IUD, approximately a third $(29.2 \%, \mathrm{n}=14)$ had their IUD for less than one year prior to their attempted self-removal. Among implant users, two women had their device for 10 months and 2 years, respectively, but the remainder did not discuss their duration of use in the video.

Table 2 presents descriptive statistics of viewer engagement with the videos included in our analysis. Collectively, these videos were uploaded between 2012-2020 and have been viewed over 4 million times. Although videos uploaded more recently have had less time for viewer engagement, the median number of views was still high at 10,473 views per video. Videos also had 68,972 likes, 6,273 viewer comments, and video creators had over 5 million subscribers. Although a much smaller proportion of videos featured the self-removal of an implant $(n=10)$, these videos had a higher average number of views (median 23,097 vs. 9,533) and comments (median 44 vs. 14) compared to videos of IUD self-removals. In contrast, videos of IUD self-removals had a higher average number of likes (74 vs. 67), and video creators who attempted IUD self-removal had more subscribers on average (98 vs. 57).

In our analysis of the top comments for each video, 3 primary themes emerged: positive affirmations, viewer's consideration or attempt of self-removal, and complaints about LARC. We provide representative quotes of user comments to illustrate each of these themes in Table 3 , along with the number of videos that received a comment from each category. Twenty-eight videos $(48.2 \%)$ included a positive affirmation for the video creator. These comments often had an encouraging tone and reflected the viewer's gratitude for the information provided. The secondmost common theme to arise from comments was the viewer's own intentions and experiences of self-removal. Twenty-five videos (43.1\%) included a comment from a viewer that stated they had 
either removed their own LARC device after watching the video or intended to do so soon. Finally, 19 videos $(32.8 \%)$ had a viewer comment that complained about their method of LARC. These comments most often expressed frustration with negative side effects or barriers to removal.

In Table 4, we highlight the primary themes that arose from our qualitative analysis of video creators' reasons for LARC discontinuation and self-removal as well as their experiences of selfremoval. Across the sample, negative side effects (both actual and potential) was the most commonly-cited reason video subjects wished to discontinue their method of LARC. A majority of IUD users $(64.6 \%, n=31)$ were motivated to discontinue due to reported side effects; in order of most common to least, these included cramping, emotional changes, weight gain, physical discomfort (e.g. pain during sex), and irregular bleeding. Although fewer implant users provided their reasons for discontinuation, the largest proportion feared potential side effects $(20 \%, n=2)$. Among those who feared potential side effects, IUDs and implants were often described as "unnatural" or a "foreign object" in the body. Other fears included artificial hormones, inflammation in the uterus, copper toxicity from the copper IUD, and reductions in fertility. Ten women (17.2\%, 1 implant user and 9 IUD users) wished to discontinue LARC in order to become (or remain) pregnant; one woman was already pregnant and wanted to discontinue an implant that a medical provider refused to remove.

We found three main motivations for LARC self-removal among video subjects. Roughly half of our sample $(n=30,51.7 \%)$ described a desire to remove their method at home out of personal preference or convenience $(58.3 \%$ of IUD users and $20 \%$ of implant users). These individuals, including 2 women whose partners removed their contraceptive implant at home, framed removal as a simple process, saying "When they do this [remove the implant] at the doctor, they do the exact same thing" and "They don't do anything special when you go to the doctor. They just pull it [the IUD] out." Another woman felt comfortable with self-removal because her provider 
left the strings long and counseled her on how to remove it. Others noted the inconvenience of an in-clinic removal and said that self-removal "saved me a trip to the doctor". Several creators, upon deciding to discontinue, found other people's stories online and were encouraged to attempt their own removal. In comparison to preference-based motivations for self-removal, a large proportion of video creators described barriers to clinic-based removal, including cost or lack of insurance $(31.3 \%$ of IUD users and $40 \%$ of implant users) and long waiting times for an appointment $(22.9 \%$ of IUD users).

Most video creators were successful in removing their own LARC device at home $(\mathrm{n}=56$, 96.6\%). Experiences of IUD removal were most often described in positive terms, such as "quick and easy" or "painless". Although 1 implant user was unable to remove the device after 20 minutes, the remainder $(n=9)$ successfully removed their implants at home. Most video creators $(n=36$, 62.1\%) removed their device on their own, without any support or assistance from another person, however, this varied dramatically according to the method. Ninety percent $(n=9)$ of implant users had a support person that removed their device, compared to just $27 \%$ of IUD users $(n=13)$.

Most videos with implant users reflected positive experiences, with individuals expressing happiness following the removal or surprise related to the low level of pain. Although most videos reflected a positive experience of self-removal, we identified roughly a third of all video creators who encountered challenges, including difficulty grasping the strings of their IUD or challenges removing the implant $(29.2 \%$ of IUD users and $30 \%$ of implant users).

\section{Discussion}

We analyzed videos created by a self-selecting group—individuals who removed their LARC devices at home and shared a video about their experience online. Although this data source is novel, we are unable to draw conclusions about the population prevalence of LARC self-removal, 
disparities in access to clinic-based removal, or how preferences for self-removal may vary across demographic groups. Individuals who fail to remove their device or have negative experiences of attempted self-removal may be less likely to share their stories online. Our goal, however, was not to describe the experience of all people who attempt self-removal, but rather to explore narratives of LARC self-removal on an accessible and widely used video-sharing website. As the first content analysis of online videos describing LARC self-removal, this study provides important data about the characteristics, motivations, and experiences of a group of people that are often invisible to researchers and healthcare providers. With more than 4 million views, the videos in our sample represent an important source of information for women who wish to discontinue LARC and, whether due to preferences or barriers, seek information about self-removal.

We hope that these findings will motivate further research exploring prevalence and demographic differences in LARC self-removal. Studies of self-managed abortion may offer promising pathways for recruitment and analysis. Such studies have surveyed clinic patients about their experiences attempting to induce an abortion prior to seeking formal care [30]; recruited participants in community settings and via snowball sampling [31]; and conducted populationrepresentative phone-based surveys [32,33]. Future studies using similar methods are needed to measure prevalence, interest, and outcomes related to LARC self-removal.

By shedding light on mainstream narratives of LARC self-removal, this study contributes further evidence of the tension between the growing prevalence of LARC use and reproductive autonomy. Nationally representative estimates from 2011-2015 found that, compared to white and Hispanic women, a significantly smaller percentage of Black women (7\%) are current LARC users [28]. Despite this lower proportion of LARC users, Black women made up almost a third of video creators in our sample. This may reflect a higher prevalence of LARC self-removal and is consistent with previous research which suggests that autonomous control may be an especially important 
feature of contraceptive method preference among Black women $[11,22,29]$. Black women may also be more likely to use online platforms such as YouTube for the exchange of health-related information, particularly since Black LARC users tend to be younger than their white counterparts [28]. While we are unable to disentangle the factors that may have led to the overrepresentation of Black women in our sample, our findings inform strategies for contraceptive counseling and provision that may contribute to reproductive justice and mitigate any risks associated with selfremoval.

Racial and ethnic differences in contraceptive attitudes and preferences are important to consider given a history of racist and coercive family planning practices in the United States. For example, the first large-scale human trial of oral contraceptives was carried out among Puerto Rican women in the 1950's and failed to provide full informed consent of the experimental nature and risks of the drug in its early stages [24]. Similarly, when Depo Provera was first developed in the 1990s, it was framed as a solution to an alleged overpopulation of women of color in poverty, and physicians were encouraged to suggest it to their patients of color [12]. Today, researchers and healthcare providers have developed interventions and targeted strategies to increase LARC use among Black, Indigenous, and Latina women, groups deemed as "high-risk" for unintended pregnancy $[22,25]$. Due to this history of exploitation and contemporary trends toward coercion, attitudes towards contraception and the medical establishment often differ by race and ethnicity $[26,27]$. We offer recommendations for clinicians and policy makers to reduce the risk of harm among those who face structural barriers to formal LARC removal and improve the patient-provider relationship, two goals that will have a disproportionate impact on people of color. In doing so, we join with researchers who call for a people-first rather than LARC-first approach to contraceptive provision and counseling [22,23]. 
Overall, video subjects' decisions to self-remove were shaped by both preferences and barriers to clinic-based care. Most video creators who cited financial barriers only discovered high costs or insurance requirements upon wanting to discontinue LARC. Previous research reveals that this is not an isolated concern [34]. We support the elimination of all out-of-pocket costs associated with LARC removal, however, in absence of such a policy, providers could lend greater support to women's reproductive autonomy by clearly describing any procedural or financial requirements of removal prior to the insertion of LARC.

Most users were successful in removing their device and portrayed self-removal in positive terms. Proactive discussion of the risks and best practices for the safe home-removal of IUDs could be incorporated into contraceptive counseling. This could include a conversation between the patient and provider about the desired length of the IUD strings. Such discussions would likely improve patient-provider trust and enhance reproductive autonomy. 
Table 1: Characteristics of YouTube video creators who removed their own intrauterine device or implant at home $(n=58)$

\begin{tabular}{|c|c|c|c|c|c|c|}
\hline \multirow[b]{2}{*}{ Video Creators } & \multirow[b]{2}{*}{ Total } & \multirow[b]{2}{*}{$\%$} & \multicolumn{4}{|c|}{ Contraceptive method } \\
\hline & & & IUD & col \% & Implant & $\operatorname{col} \%$ \\
\hline \multicolumn{7}{|l|}{ Gender } \\
\hline Woman & 57 & 98.3 & 47 & 97.9 & 10 & 100.0 \\
\hline Non-binary & 1 & 1.7 & 1 & 2.1 & 0 & 0.0 \\
\hline \multicolumn{7}{|l|}{ Age group } \\
\hline$<20$ & 3 & 5.2 & 2 & 4.2 & 1 & 10.0 \\
\hline $20-29$ & 39 & 67.2 & 32 & 66.7 & 7 & 70.0 \\
\hline $30-39$ & 14 & 24.1 & 14 & 29.2 & 1 & 10.0 \\
\hline 40 or older & 2 & 3.4 & 2 & 4.2 & 0 & 0.0 \\
\hline Unknown & 0 & 0 & 0 & 0.0 & 1 & 10.0 \\
\hline \multicolumn{7}{|l|}{ Race/ethnicity } \\
\hline White & 31 & 53.4 & 24 & 50.0 & 6 & 60.0 \\
\hline Black & 18 & 31.0 & 16 & 33.3 & 2 & 20.0 \\
\hline Latina & 8 & 13.8 & 7 & 14.6 & 1 & 10.0 \\
\hline Native American & 1 & 1.7 & 1 & 2.1 & 0 & 0.0 \\
\hline Unknown & 0 & 0.0 & 0 & 0.0 & 1 & 10.0 \\
\hline \multicolumn{7}{|l|}{ Previous childbearing } \\
\hline Has children & 22 & 37.9 & 19 & 39.6 & 3 & 30.0 \\
\hline No children / not discussed & 36 & 62.1 & 29 & 60.4 & 7 & 70.0 \\
\hline \multicolumn{7}{|l|}{ Type of IUD } \\
\hline ... hormonal & & & 34 & 70.8 & & \\
\hline ... copper & & & 14 & 29.2 & & \\
\hline \multicolumn{7}{|c|}{$\begin{array}{l}\text { Duration of LARC use prior to } \\
\text { attempted self-removal }\end{array}$} \\
\hline$<6$ months & 8 & 13.8 & 8 & 16.7 & 0 & 0.0 \\
\hline 6 months -1 year & 7 & 12.1 & 6 & 12.5 & 1 & 10.0 \\
\hline $1-5$ years & 26 & 44.8 & 25 & 54.2 & 1 & 10.0 \\
\hline$>5$ years & 3 & 5.2 & 3 & 6.3 & 0 & 0.0 \\
\hline Not discussed & 14 & 24.1 & 6 & 12.5 & 8 & 80.0 \\
\hline Total & 58 & & 48 & & 10 & \\
\hline
\end{tabular}

Note: Percentages may not sum to 100 due to rounding. 
Table 2: Viewer engagement with YouTube videos portraying the self-removal of long-acting reversible contraceptive methods $(\mathrm{n}=58)$

\begin{tabular}{llllllllll}
\hline $\begin{array}{c}\text { Viewer } \\
\text { Engagement }\end{array}$ & Total & Median & Range & IUD & Median & Range & Implant & Median & Range \\
\hline Views & $4,237,349$ & 10,473 & $23: 1,911,945$ & $1,999,986$ & 9,533 & $23: 454,167$ & 2188731 & 23097 & $267: 1,911,945$ \\
Likes $^{\dagger}$ & 68,972 & 81 & $0: 46,000$ & 21,808 & 74 & $0: 8,000$ & 46,540 & 67 & $4: 46,000$ \\
Comments $^{\dagger 1}$ & 6,273 & 16 & $0: 3,138$ & 2,629 & 14 & $0: 445$ & 3644 & 44 & $1: 3,138$ \\
Subscribers & $5,059,371$ & 84 & $0: 3,200,000$ & 951745 & 98 & $0: 849,000$ & 3210426 & 57 & $15: 3,210,000$ \\
\hline
\end{tabular}

\footnotetext{
${ }^{\dagger}$ The likes/dislikes feature was disabled for 1 video and is not included

${ }^{11} 4$ video creators disabled comments and 9 videos had no comments
} 
Table 3: Illustrative quotes of viewer comments from 45 YouTube videos, by method of LARC and theme ${ }^{\dagger}$

IUD self-removal videos

Implant self-removal videos

Positive affirmations $(n=28)$

$(\mathrm{n}=21)$

$(n=7)$

Short, sweet, and straight to the point! This is the

most helpful video I have watched about removing

the iud

Quick and clean, Good Job!

I appreciate your video because I am honestly

I will pay this person to please do mine.

contemplating pulling it out myself.

Considered or attempted self-removal of LARC (n=25)

$(\mathrm{n}=20)$

$(\mathrm{n}=5)$

Thank you so much! I watched other videos and I just removed mine today. 2 hours myself.

tried for like an hour and I watched this one and I had to cut really deep in the tissue but got once you said to use the thumb and pointer finger I it and couldn't be happier :)

got it out! I'm so empowered and happy!

Watched your video. Went to bathroom. Came out iud free.

Complaints about LARC $(n=19)$

$(n=14)$

$(\mathrm{n}=5)$

Watching this video made me feel so understood.

I've had my IUD now for approximately 3 years ...

Please tell me how to do this, i want this

Getting my IUD inserted was by far one of the out of me like TOMORROW! And i'm so most terrifyingly painful experiences of my life. The pain was literally just as you described it. I also almost passed out from the pain. The whole experience was honestly scaring.

I got my Mirena in December 2014 and have gained 20 pounds, had crazy carb cravings, felt really bloated in the belly. Went to the doctor in April 2016 to take it out but they talked me out of it, saying the weight gain was due to getting old. well, I spent three next month making a real conscious effort to lose weight and ended up gaining weight, so I went back in last week and had it removed. I feel great.

I've been bleeding for almost 4 months. [The implant] does a great job with keeping me not pregnant, but the stress acne, horrible cramps and depressive episodes are NOT worth it!
Doing this this week. This thing is the devil!
If I would've know it was this easy I would've taken it out, I went from weighing 165 to 210 and I was eating all my normal foods, then I tried diets and cleanses but nothing worked for me and I got really depressed about my weight so thanks for this video

Literally bought all the stuff to do this on Amazon because the effects of it that is has on my body are not great :(

${ }^{\dagger} 4$ video creators disabled comments and 9 videos had no user comments 
Table 4: Motivations and experiences of video contributors who removed a long-acting reversible contraceptive device at home $(\mathrm{N}=58)$

\begin{tabular}{ccccc} 
Total $\%$ & \multicolumn{3}{c}{ Method of LARC } \\
\hline & IUD & $\%$ IUD & Implant & $\%$ implant \\
& $(\mathrm{n}=48)$ & users & $(\mathrm{n}=10)$ & users \\
\hline
\end{tabular}

\section{$\underline{\text { Reasons for LARC discontinuation }}$}

Desired pregnancy

$10 \quad 17.2$

Negative side effects

$32 \quad 55.2$

9

18.8

1

10.0

Fear of potential side effects

$15 \quad 25.9$

31

64.6

1

10.0

$\underline{\text { Reasons for self-removal of LARC }}$

$\begin{array}{lllllll}\text { Cost/ lack of insurance } & 19 & 32.8 & 15 & 31.3 & 4 & 40.0 \\ \text { Waiting time for an appointment } & 11 & 19.0 & 11 & 22.9 & 0 & 0.0 \\ \text { Preference/ Convenience } & 30 & 51.7 & 28 & 58.3 & 2 & 20.0\end{array}$

\section{Experience of LARC self-removal}

$\begin{array}{lcccccc}\text { Successful removal } & 56 & 96.6 & 47 & 97.9 & 9 & 90.0 \\ \text { Easy } & 38 & 65.6 & 35 & 72.9 & 3 & 30.0 \\ \text { Little pain } & 36 & 62.1 & 32 & 66.7 & 4 & 40.0 \\ \text { Had support person for removal } & 22 & 37.9 & 13 & 27.1 & 9 & 90.0 \\ \begin{array}{l}\text { Difficult (time, grasping IUD strings } \\ \text { or implant, etc.) }\end{array} & 17 & 29.3 & 14 & 29.2 & 3 & 30.0\end{array}$

Note: themes within each category are not mutually exclusive. For example, a video subject may have been motivated to discontinue LARC because they desired pregnancy and they experienced negative side effects. 
Appendix A: Keyword search terms used to produce a comprehensive list of YouTube videos related to the self-removal of LARC

Desired video content

Keyword search

IUD self-removal (iud OR mirena OR skyla OR kyleena OR liletta OR paragard) AND ("self-removal" OR diy OR home)

Implant self-removal (implant OR nexplanon OR implanon) AND ("self-removal" OR DIY OR home) 


\section{References}

[1] Finer LB, Jerman J, Kavanaugh ML. Changes in use of long-acting contraceptive methods in the United States, 2007-2009. Fertil Steril 2012;98:893-7. https://doi.org/10.1016/j.fertnstert.2012.06.027.

[2] Kavanaugh ML, Jerman J. Contraceptive method use in the United States: trends and characteristics between 2008, 2012 and 2014. Contraception 2018;97:14-21. https://doi.org/10.1016/j.contraception.2017.10.003.

[3] Sobel L, Salganicoff A, Frederiksen B. New Title X Regulations: Implications for Women and Family Planning Providers. Kais Fam Found 2019.

[4] Jones RK, Witwer E, Jerman J. Abortion Incidence and Service Availability in the United States, 2017 2019. https://doi.org/10.1363/2019.30760.

[5] White K, Hopkins K, Aiken ARA, Stevenson A, Hubert C, Grossman D, et al. The Impact of Reproductive Health Legislation on Family Planning Clinic Services in Texas. Am J Public Health 2015;105:851-8. https://doi.org/10.2105/AJPH.2014.302515.

[6] Lindbergh L, VandeVusse A, Mueller J, Kirstein M. Early Impacts of the COVID-19 Pandemic: Findings from the 2020 Guttmacher Survey of Reproductive Health Experiences. Guttmacher Institute; 2020.

[7] Senderowicz L, Higgins J. Reproductive Autonomy Is Nonnegotiable, Even in the Time of COVID-19. Perspect Sex Reprod Health 2020;52:81-5. https://doi.org/10.1363/psrh.12152.

[8] ACOG. COVID-19 FAQs for Obstetrician-Gynecologists, Gynecology 2021. https://www.acog.org/en/clinical-information/physician-faqs/covid19-faqs-for-ob-gynsgynecology (accessed April 26, 2021).

[9] Kaunitz AM. COVID-19 Gynecology Practice Recommendations from ACOG. NEJM J Watch Womens Health 2020.

[10] Amico JR, Stimmel S, Hudson S, Gold M. “\$231 ... to pull a string!!!” American IUD users’ reasons for IUD self-removal: An analysis of internet forums. Contraception 2020;101:393-8. https://doi.org/10.1016/j.contraception.2020.02.005.

[11] Foster DG, Grossman D, Turok DK, Peipert JF, Prine L, Schreiber CA, et al. Interest in and experience with IUD self-removal. Contraception 2014;90:54-9. https://doi.org/10.1016/j.contraception.2014.01.025.

[12] Roberts D. Killing the Black Body: Race, Reproduction, and the Meaning of Liberty. New York: Vintage Books; 1998.

[13] Heilman JM, West AG. Wikipedia and Medicine: Quantifying Readership, Editors, and the Significance of Natural Language. J Med Internet Res 2015;17. https://doi.org/10.2196/jmir.4069.

[14] Vogel L. Dr. YouTube will see you now. Can Med Assoc J CMAJ Ott 2011;183:647-8.

[15] Ache KA, Wallace LS. Human Papillomavirus Vaccination Coverage on YouTube. Am J Prev Med 2008;35:389-92. https://doi.org/10.1016/j.amepre.2008.06.029.

[16] Agrawal S, Irwin C, Dhillon-Smith RK. An evaluation of the quality of online information on emergency contraception. Eur J Contracept Reprod Health Care 2021;0:1-6. https://doi.org/10.1080/13625187.2021.1887476.

[17] Jerman J, Onda T, Jones RK. What are people looking for when they Google "self-abortion"? Contraception 2018. https:// doi.org/10.1016/j.contraception.2018.02.006.

[18] Nguyen BT, Allen AJ. Social media and the intrauterine device: a YouTube content analysis. BMJ Sex Reprod Health 2018;44:28-32. https://doi.org/10.1136/bmjsrh-2017-101799. 
[19] Paul J, Boraas CM, Duvet M, Chang JC. YouTube and the single-rod contraceptive implant: a content analysis. J Fam Plann Reprod Health Care 2017;43:195-200. https://doi.org/10.1136/jfprhc-2016-101593.

[20] Weiss E, Moore K. An assessment of the quality of information available on the internet about the IUD and the potential impact on contraceptive choices. Contraception 2003;68:359-64. https://doi.org/10.1016/j.contraception.2003.07.001.

[21] Raifman S, Barar R, Foster D. Effect of Knowledge of Self-removability of Intrauterine Contraceptives on Uptake, Continuation, and Satisfaction. Womens Health Issues 2018;28:6874. https://doi.org/10.1016/j.whi.2017.07.006.

[22] Gomez AM, Fuentes L, Allina A. Women or LARC First? Reproductive Autonomy and the Promotion of Long-Acting Reversible Contraceptive Methods. Perspect Sex Reprod Health 2014;46:171-5. https://doi.org/10.1363/46e1614.

[23] Higgins JA, Kramer RD, Ryder KM. Provider Bias in Long-Acting Reversible Contraception (LARC) Promotion and Removal: Perceptions of Young Adult Women. Am J Public Health 2016;106:1932-7. https://doi.org/10.2105/AJPH.2016.303393.

[24] Briggs L. Reproducing Empire. 1st ed. University of California Press; 2003.

[25] Mann ES, Grzanka PR. Agency-Without-Choice: The Visual Rhetorics of Long-Acting Reversible Contraception Promotion. Symb Interact 2018;41:334-56. https://doi.org/10.1002/symb.349.

[26] Rocca CH, Harper CC. Do Racial and Ethnic Differences in Contraceptive Attitudes and Knowledge Explain Disparities In Method Use? Perspect Sex Reprod Health 2012;44:150-8. https://doi.org/10.1363/4415012.

[27] Thorburn S, Bogart LM. Conspiracy Beliefs About Birth Control: Barriers to Pregnancy Prevention Among African Americans of Reproductive Age. Health Educ Behav 2005;32:47487. https://doi.org/10.1177/1090198105276220.

[28] Kramer RD, Higgins JA, Godecker AL, Ehrenthal DB. Racial and ethnic differences in patterns of long-acting reversible contraceptive use in the United States, 2011-2015. Contraception 2018;97:399-404. https://doi.org/10.1016/j.contraception.2018.01.006.

[29] Jackson AV, Karasek D, Dehlendorf C, Greene Foster D. Racial and ethnic differences in women's preferences for features of contraceptive methods. Contraception 2016;93:406-11.

[30] Aiken ARA, Broussard K, Johnson DM, Padron E, Starling JE, Scott JG. Knowledge, interest, and motivations surrounding self-managed medication abortion among patients at three Texas clinics. Am J Obstet Gynecol 2020;223. https://doi.org/10.1016/j.ajog.2020.02.026.

[31] Fuentes L, Baum S, Keefe-Oates B, White K, Hopkins K, Potter J, et al. Texas women's decisions and experiences regarding self-managed abortion. BMC Womens Health 2020;20:112. https://doi.org/10.1186/s12905-019-0877-0.

[32] Grossman D, Hendrick E, Fuentes L, White K, Hopkins K, Stevenson A, et al. Knowledge, opinion and experience related to abortion self-induction in Texas. Contraception 2015;92:360-1. https://doi.org/10.1016/j.contraception.2015.06.036.

[33] Grossman D, Ralph L, Raifman S, Upadhyay U, Gerdts C, Biggs A, et al. Lifetime prevalence of self-induced abortion among a nationally representative sample of U.S. women. Contraception 2018;97:460. https://doi.org/10.1016/j.contraception.2018.03.017.

[34] Amico JR, Heintz C, Bennett AH, Gold M. Access to IUD removal: Data from a mysterycaller study. Contraception 2020;101:122-9. https://doi.org/10.1016/j.contraception.2019.10.008. 